\title{
A20 prevents inflammasome-dependent arthritis by inhibiting macrophage necroptosis through its $\mathrm{ZnF7}$ ubiquitin binding domain
}

Apostolos Polykratis ${ }^{1, \#}$, Arne Martens ${ }^{2,3, \#, ~ R e m z i ~ O n u r ~ E r e n ~}{ }^{1, \#}$, Yoshitaka Shirasaki ${ }^{4,} 5$, Mai Yamagishi ${ }^{5}$, Yoshifumi Yamaguchi ${ }^{6,}{ }^{7}$, Sotaro Uemura ${ }^{5}$, Masayuki Miura ${ }^{6}$, Bernhard Holzmann ${ }^{8}$, George Kollias $^{9,10}$, Marietta Armaka, ${ }^{9,}$, Geert van Loo ${ }^{2,3, \S, *}$, Manolis Pasparakis ${ }^{1, \S, *}$.

${ }^{1}$ Institute for Genetics, Cologne Excellence Cluster on Cellular Stress Responses in AgingAssociated Diseases (CECAD) \& Center for Molecular Medicine (CMMC), University of Cologne, Cologne, Germany

${ }^{2}$ VIB Center for Inflammation Research, Ghent B-9052, Belgium

${ }^{3}$ Department of Biomedical Molecular Biology, Ghent University, Ghent B-9052, Belgium

${ }^{4}$ JST PRESTO, Japan

${ }^{5}$ Department of Biological Sciences, Graduate School of Science, The University of Tokyo, Japan

${ }^{6}$ Department of Genetics, Graduate School of Pharmaceutical Science, The University of Tokyo, Japan

${ }^{7}$ Institute of Low Temperature Science, Hokkaido University, Japan

${ }^{8}$ Technical University of Munich, School of Medicine, Department of Surgery, Ismaninger Str. 22, 81675 Munich, Germany

${ }^{9}$ Biomedical Sciences Research Center "Alexander Fleming", 16672 Vari, Greece

${ }^{10}$ Department of Physiology, Medical School, National and Kapodistrian University of Athens, 11527 Athens, Greece

\#Equal first authors

§Equal last authors

*Correspondence: G.v.L. (geert.vanloo@irc.vib-ugent.be), M.P. (pasparakis@uni-koeln.de)

Published in Nat Cell Biol 2019, 21(6):731-742

doi: 10.1038/s41556-019-0324-3 


\begin{abstract}
Deficiency in the deubiquitinating enzyme A20 causes severe inflammation in mice and impaired A20 function is associated with human inflammatory diseases. A20 has been implicated in negatively regulating NF-KB signalling, cell death and inflammasome activation, however the mechanisms by which A20 inhibits inflammation in vivo remain poorly understood. Genetic studies in mice revealed that its deubiquitinase activity is not essential for A20 anti-inflammatory function. Here we show that A20 prevents inflammasomedependent arthritis by inhibiting macrophage necroptosis and that this function depends on its zinc finger 7 (ZnF7). We provide genetic evidence that RIPK1 kinase-dependent, RIPK3MLKL-mediated necroptosis drives inflammasome activation in A20-deficient macrophages and causes inflammatory arthritis in mice. Single cell imaging revealed that RIPK3dependent death caused inflammasome-dependent IL-1 $\beta$ release from LPS-stimulated A20deficient macrophages. Importantly, mutation of the A20 ZnF7 ubiquitin binding domain caused arthritis in mice, arguing that $\mathrm{ZnF7}$-dependent inhibition of necroptosis is critical for A20 anti-inflammatory function in vivo.
\end{abstract}

A20 (also known as TNF inducible protein 3, TNFAIP3) is a deubiquitinating enzyme with critical anti-inflammatory functions. Genome wide association studies (GWAS) identified A20 as a susceptibility gene in several human inflammatory and autoimmune diseases, including psoriasis, systemic lupus erythematosus (SLE), Crohn's disease and rheumatoid arthritis (reviewed in ${ }^{1-4}$ ). Moreover, mutations in the $A 20$ gene have been identified in B cell lymphoma patients ${ }^{5-8}$. More recently, $A 20$ haploinsufficiency was identified as the cause of early-onset autoinflammatory and autoimmune conditions ${ }^{9-13}$. Studies in genetic mouse models supported a key role for A20 as an inhibitor of inflammation. Depending on the genetic background, A20-deficient mice die perinatally or in the first weeks of life due to severe lethal multi-organ inflammation ${ }^{14-16}$. Moreover, tissuespecific A20 deficiency sensitized mice to inflammatory and autoimmune pathologies ${ }^{17-26}$. While its important anti-inflammatory role in both humans and mice is indisputable, the mechanisms by which A20 suppresses inflammation in vivo remain poorly understood.

Inhibition of NF- $\kappa B$ activation is considered the primary anti-inflammatory function of $A 20\left({ }^{1,2,27,28}\right)$. In addition, A20 was also suggested to inhibit necroptosis by deubiquitinating RIPK3(15). Although receptor interacting protein kinase 3 (RIPK3) deficiency prolonged survival of A20 knockout mice, mixed lineage kinase-like (MLKL) deficiency did not have any protective effect arguing against a role for necroptosis in the pathology caused A20 deficiency ${ }^{15}$, ${ }^{16}$. Degradation of lysine 63 (K63)linked ubiquitin chains by A20 is considered important for inhibition of inflammation by preventing the ubiquitin-dependent recruitment of the inhibitor of NF-kB kinase (IKK) and transforming growth factor beta-activating kinase 1 (TAK1) signalling complexes to upstream receptors ${ }^{1,2,27-29}$. In addition, the ZnF4 domain of A20 was proposed to limit tumour necrosis factor receptor 1 (TNFR1) signalling by mediating K48-linked ubiquitination of RIPK1(29). However, knock-in mice expressing A20 with mutated catalytic ovarian tumor (OTU) or ZnF4 domains did not develop spontaneous inflammatory pathology, arguing that ubiquitin chain degradation and ZnF4-dependent functions are not essential for A20-mediated suppression of inflammation in vivo ${ }^{28,30,31}$. The capacity to bind to ubiquitin chains through its $\mathrm{ZnF}$ domains 4 and 7 has also been implicated in A20 function ${ }^{32-36}$. In particular, the ZnF7 of A20 was implicated in regulating TNFR1 signalling by binding specifically to linear ubiquitin chains ${ }^{28,37}$ and inhibiting TNFR1-mediated apoptosis and necroptosis ${ }^{35,37}$. However, the in vivo function of the $\mathrm{A} 20 \mathrm{ZnF7}$ remains unknown.

We showed previously that mice with myeloid cell specific A20 deficiency (A20 ${ }^{\mathrm{MYC}-\mathrm{KO}}$ ) spontaneously develop inflammatory joint pathology resembling rheumatoid arthritis ${ }^{23}$. Arthritis development in A20 ${ }^{\mathrm{MYC}-\mathrm{KO}}$ mice requires NLR Family Pyrin Domain Containing 3 (NLRP3), caspase-1 and interleukin 1 receptor (IL-1R1) signalling, suggesting that regulation of inflammasome activation is a key anti-inflammatory function of $\mathrm{A} 20(23,38)$. However, the mechanisms by which A20 limits inflammasome activation remain poorly understood. Excessive inflammasome priming through impaired A20-dependent negative regulation of NF-kB-mediated expression of NLRP3 and pro-IL- 
$1 \beta$ was suggested to contribute to inflammasome hyperactivation and arthritis development based on in vitro studies using macrophages from $\mathrm{A} 2 \mathrm{O}^{\mathrm{MYC}-\mathrm{KO}}$ mice $^{38}$. In addition, $\mathrm{A} 20$ was reported to negatively regulate ubiquitination of pro-IL-1 $\beta$ as well as components of the NLRP3/Caspase-1 inflammasome in a RIPK3-dependent but cell death-independent manner, suggesting that A20 directly regulates inflammasome activity by deubiquitination ${ }^{39}$. Therefore, the pathways that are controlled by $\mathrm{A} 20$ and are critical for inhibition of inflammation in vivo as well as the functional domains of $\mathrm{A} 20$ that regulate these activities remain elusive.

Here we provide genetic and biochemical evidence that A20 prevents inflammasome activation and arthritis pathology indirectly by inhibiting macrophage necroptosis. Moreover, we show that knock-in mice expressing A20 with mutated ZnF7 develop spontaneous arthritis, revealing an indispensable function of ZnF7-mediated binding to linear ubiquitin chains in A20-mediated suppression of inflammation in vivo. Together, these results identify inhibition of necroptosis as a critical antiinflammatory function of A20 in vivo and suggest that necroptosis inhibitors could be effective for the treatment of arthritis and other inflammatory pathologies associated with impaired A20 activity.

\section{Results}

Inflammasome activation in myeloid cells causes arthritis in $\mathrm{A20}^{\mathrm{MYC}-\mathrm{KO}}$ mice.

$\mathrm{A} 20^{\mathrm{MYC}-\mathrm{KO}}$ mice spontaneously develop inflammatory joint pathology resembling rheumatoid arthritis, characterised by immune cell infiltration, bone erosion and cartilage destruction, and show splenomegaly and elevated numbers of blood monocytes and neutrophils ${ }^{23}$ (Figure 1a-c and

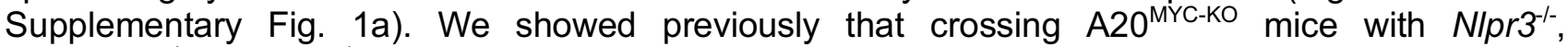
Casp $1 / 11^{-/-}$or $I I-1 r 1^{-1-}$ mice strongly ameliorated arthritis development, suggesting that NLRP3 inflammasome-mediated IL-1 $\beta$ production drives the pathology ${ }^{23}, 38$. To assess if cell-intrinsic inflammasome activation in A20-deficient myeloid cells drives arthritis, we generated mice lacking both A20 and apoptosis-associated speck-like protein containing CARD (ASC), an adapter required for the recruitment and activation of caspase-1 downstream of NLRP3 (40), specifically in myeloid cells. ASC ablation reduced the levels of IL-1 $1, \mathrm{IL}-18$ and TNF in the serum and ameliorated splenomegaly in A20 ${ }^{\mathrm{MYC}-\mathrm{KO}}$ mice (Fig. $1 \mathrm{a}, \mathrm{b}$ and Supplementary Fig. 1b). Myeloid-specific ASC deficiency also substantially reduced arthritis severity but could not fully prevent joint inflammation as well as bone and cartilage damage (Figure 1c-h). LPS stimulation triggered ASC-dependent IL$1 \beta$ release from A20-deficient bone marrow-derived macrophages (BMDMs) as shown previously ${ }^{39}$, but also caused ASC-independent IL-1 $\alpha$ release (Figure 1i), suggesting that inflammasomeindependent IL-1 $\alpha$ production could also contribute to the development of arthritis.

\section{Arthritis development in A20 ${ }^{\mathrm{MYC}-\mathrm{KO}}$ mice depends on MyD88 signalling in both myeloid and non-myeloid cells.}

We showed previously that MyD88 deficiency prevented the development of arthritis, splenomegaly and myeloid cell expansion in A20 ${ }^{\mathrm{MYC}-\mathrm{KO}}$ mice $^{23}$. To investigate the relative contribution of MyD88 signalling in myeloid- and non-myeloid cells we employed a dual approach. First, we generated mice lacking both $\mathrm{A} 20$ and MyD88 in myeloid cells $\left(\mathrm{A} 20^{\mathrm{MYC}-\mathrm{KO}} \mathrm{MyD}^{\mathrm{MYC}-\mathrm{KO}}\right.$ ) and found that myeloid cell-specific MyD88 ablation prevented arthritis, suppressed splenomegaly and myeloid cell expansion, normalised IL-1 $\beta$ and reduced TNF levels, but did not significantly affect IL-18, IL-6, and MCP-1 production in A20 ${ }^{\text {MYC-KO }}$ mice (Fig. 2a-h and Supplementary Fig. 1c,d). MyD88 deficiency also strongly reduced the LPS-induced release of $\mathrm{IL}-1 \beta$ and $\mathrm{IL}-1 \alpha$ from A20-deficient BMDMs, which correlated with reduced transcription of the il1b and il1a mRNAs (Figure $2 \mathrm{i}-\mathrm{j}$ ). To assess the role of MyD88 in non-myeloid cells we employed Myd88 ${ }^{\mathrm{LSL}}$ mice carrying Myd88 alleles containing a loxP-flanked transcriptional stop cassette in intron 1, which do not express MyD88 unless the loxPflanked stop cassette is excised by Cre recombinase ${ }^{41}$. By intercrossing $\mathrm{A} 2 \mathrm{O}^{\mathrm{MYC}-\mathrm{KO}}$ mice with $M y d 88^{\mathrm{LSL}}$ animals we obtained mice with myeloid cells that lack $\mathrm{A} 20$ but express MyD88, while nonmyeloid cells express A20 but lack MyD88 (A20 ${ }^{\mathrm{MYC}-\mathrm{KO}}$ MyD88 $^{\mathrm{MYC}}$ ). MyD88 deficiency in non-myeloid cells did not inhibit monocytosis but partly reduced neutrophilia and strongly suppressed splenomegaly in $\mathrm{A} 2 \mathrm{O}^{\mathrm{MYC}-\mathrm{KO}}$ mice (Fig. 2a and Supplementary Fig. 1c). Importantly, A20 ${ }^{\mathrm{MYC}-\mathrm{KO}}$ MyD88 $^{\mathrm{MYC}}$ mice were protected from arthritis and had reduced levels of IL-1 $\beta$, IL-18, TNF and IL-6 
in the serum (Fig. 2b-h and Supplementary Fig. 1d). Therefore, MyD88 acts in both myeloid and non-myeloid cells to induce the pathogenesis of inflammatory arthritis in $\mathrm{A} 20^{\mathrm{MYC}-\mathrm{KO}}$ mice.

\begin{abstract}
MyD88-dependent signalling in synovial fibroblasts contributes to the development of arthritis in A20 ${ }^{\mathrm{MYC}-\mathrm{KO}}$ mice

MyD88 signalling in myeloid cells likely regulates arthritis development by inducing the expression of IL-1 $\beta$ and IL- $1 \alpha$, however, how MyD88 acts in non-myeloid cells to drive arthritis in A20 MYC-KO mice is unclear. We postulated that MyD88 acts downstream of the IL-1 receptor in synovial fibroblasts, which are activated by IL-1 and mediate joint tissue destruction and inflammation by producing metalloproteases and inflammatory cytokines ${ }^{42,43}$, to drive arthritis pathogenesis. We therefore generated mice lacking MyD88 in synovial fibroblasts (MyD88 ${ }^{\mathrm{SF}-\mathrm{KO}}$ ) by crossing Myd88 $8^{\mathrm{FL} / \mathrm{FL}}$ with Col6a1Cre transgenic mice ${ }^{44}$. Synovial fibroblasts from MyD88 ${ }^{\mathrm{SF}-\mathrm{KO}}$ mice showed impaired NF$\kappa \mathrm{B}$ and mitogen activated protein kinase (MAPK) activation and strongly reduced expression of cytokines and metalloproteinases in response to stimulation with LPS or IL-1 $\beta$ (Supplementary Fig. $2 a-c)$. To address the role of MyD88 in synovial fibroblasts in the development of arthritis, we

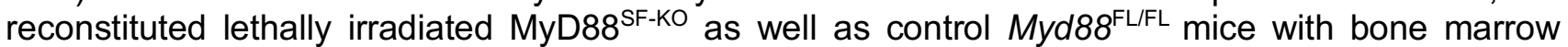
from $\mathrm{A} 20^{\mathrm{MYC}-\mathrm{KO}}$ or littermate $A 20^{\mathrm{FL} / \mathrm{FL}}$ mice. MyD88 ${ }^{\mathrm{SF}-\mathrm{KO}}$ recipients that received $\mathrm{A} 20^{\mathrm{MYC}-\mathrm{KO}}$ bone marrow showed reduced arthritis and splenomegaly compared to wild type recipients, while monocytosis and neutrophilia were not affected (Fig. 3, Supplementary Fig. 2d). MyD88 acts downstream of TLRs as well as IL-1 family receptors. Our earlier work provided evidence that both TLR4 and IL-1R signalling are important for the pathogenesis of arthritis in A20 ${ }^{\mathrm{MYC}-\mathrm{KO}}$ mice $^{23}$. We found that synovial fibroblast specific knockout of TLR4 did not affect arthritis and splenomegaly induced by reconstitution with bone marrow from $\mathrm{A} 20^{\mathrm{MYC}-\mathrm{KO}}$ mice (Supplementary Fig. 3), further supporting that MyD88 acts downstream of IL-1R in synovial fibroblasts. Collectively, these results argue that activation of arthritogenic MyD88-dependent signalling in SFs of $A 20^{\mathrm{MYC}-\mathrm{KO}}$ animals occurs downstream of IL-1R activation induced by IL-1 $\beta$ and IL-1 $\alpha$ released by $A 20$-deficient macrophages.
\end{abstract}

\title{
Myeloid cell specific IKK2 deficiency does not prevent arthritis in A20 ${ }^{\text {MYC-KO }}$ mice.
}

Inhibition of NF-KB activation is considered the main anti-inflammatory function of $A 20(1,2,27,28)$. To address the role of NF-KB we generated mice lacking both $A 20$ and IKK2/IKK $\beta$ in myeloid cells. $\mathrm{A} 20^{\mathrm{MYC}-\mathrm{KO}} \mathrm{IKK} 2^{\mathrm{MYC}-\mathrm{KO}}$ mice exhibited similar spleen size, serum IL-1 $\beta$ levels as well as inflammatory joint pathology compared to $\mathrm{A} 2 \mathrm{O}^{\mathrm{MYC}-\mathrm{KO}}$ animals, showing that NF- $\mathrm{KB}$ inhibition by IKK2 knockout in myeloid cells was not sufficient to suppress inflammation caused by A20 deficiency (Fig. 4). These results argue that NF-кB-independent functions of A20 in myeloid cells are critical for preventing inflammation in vivo.

\section{RIPK1-RIPK3-MLKL-dependent necroptosis drives arthritis development in A20 ${ }^{\mathrm{MYC}-K O}$ mice.}

In addition to inhibiting NF- $\mathrm{B}, \mathrm{A} 20$ has been implicated in the regulation of RIPK3-dependent signalling ${ }^{15},{ }^{39}$. We therefore generated $\mathrm{A} 20^{\mathrm{MYC}-\mathrm{KO}}$ Ripk $3^{-/-}$mice and found that RIPK3 deficiency prevented arthritis development (Fig. 5). RIPK3 induces MLKL-dependent necroptosis but can also promote apoptosis and has been proposed to regulate inflammation independently of cell death ${ }^{45}$. To specifically address the role of necroptosis, we generated $\mathrm{A} 2 \mathrm{O}^{\mathrm{MYC}-\mathrm{KO}} \mathrm{Mlkl^{-/ }}$ mice and found that they were also strongly protected from arthritis (Fig. 5). Inhibition of RIPK1 kinase activity has been shown to efficiently block necroptosis downstream of many receptors including death receptors and $\mathrm{TLRs}^{46}$. To assess the role of RIPK1 kinase activity we crossed A20 ${ }^{\mathrm{MYC}-\mathrm{KO}}$ mice to Ripk1 $1^{\mathrm{D} 138 \mathrm{~N} / \mathrm{D} 138 \mathrm{~N}}$ knock-in mice ${ }^{47}$ expressing kinase inactive RIPK1. Lack of RIPK1 kinase activity protected A20 MYCKO mice from the development of arthritis to a similar extent as RIPK3 or MLKL deficiency (Fig. 5). Lack of RIPK1 kinase activity as well as RIPK3 or MLKL deficiency also reduced the levels of IL-1 $\beta$ and TNF in the serum of A20 ${ }^{\mathrm{MYC}-\mathrm{KO}}$ mice (Fig. 5). However, splenomegaly was largely dependent on RIPK3-MLKL-mediated necroptosis but independent of RIPK1 kinase activity, while monocytosis was dependent on RIPK1 kinase activity but not RIPK3-MLKL (Fig. 5). Taken together, these results revealed a critical role of RIPK1-RIPK3-MLKL-dependent necroptosis in driving the pathogenesis of inflammatory arthritis in A20 ${ }^{\mathrm{MYC}-\mathrm{KO}}$ mice. 


\section{RIPK3-dependent cell death is required for IL-1 $\beta$ and IL-1 $\alpha$ release by LPS-stimulated A20 knockout macrophages}

Our genetic studies showed that RIPK1-RIPK3-MLKL-induced necroptosis drives IL-1 $\beta$ release in vivo and arthritis development in $\mathrm{A} 20^{\mathrm{MYC}-\mathrm{KO}}$ mice. To address how RIPK1, RIPK3 and MLKL regulate the release of IL-1 $\beta$ from A20-deficient myeloid cells, we studied the responses of BMDMs from the different mouse lines. RIPK3 or MLKL deficiency or lack of RIPK1 kinase activity did not affect the LPS- or TNF-induced activation of NF- $\kappa B$ and MAPK signalling as well as the mRNA expression of inflammatory mediators in A20-deficient BMDMs (Supplementary Fig. 4), suggesting that RIPK1, RIPK3 and MLKL do not act by regulating NF-kB-mediated inflammatory gene transcription in A20-deficient macrophages.

RIPK3 deficiency prevented the LPS-induced release of processed IL-1 $\beta$ from A20-deficient BMDMs (Fig. 6a, b), as shown previously ${ }^{39}$. Interestingly, RIPK3 deficiency also prevented the inflammasome-independent release of IL-1 $\alpha$, which requires breach of the plasma membrane as for example during necrotic cell death ${ }^{48}$. These results suggested that RIPK3 might regulate the LPSinduced release of IL-1 $\beta$ and IL-1 $\alpha$ from A20-deficient macrophages by triggering cell death. MLKL deficiency reduced the LPS-induced production of IL-1 $\beta$ and IL-1 $\alpha$ by A20 knockout BMDMs, although it could not fully prevent the release of these cytokines as observed in RIPK3 deficient cells (Fig. 6a, b). The pan-caspase inhibitor z-VAD-fmk inhibited the release of IL-1 $\beta$ from A20deficient BMDMs, likely due to inhibition of caspase-1-dependent pro-IL-1 $\beta$ processing, while IL-1 $\alpha$ was still produced by these cells albeit to somewhat reduced levels (Fig. 6b). However, z-VAD-fmk fully prevented the release of IL-1 $\alpha$ from macrophages lacking both A20 and MLKL (Fig. 6b), suggesting that caspase-dependent cell death drives the production of IL-1 $\alpha$ from A20-deficient macrophages when MLKL-dependent necroptosis is blocked. Interestingly, inhibition of RIPK1 kinase activity either genetically in $\mathrm{A} 20^{\mathrm{MYC}-\mathrm{KO}}$ Ripk1 ${ }^{\mathrm{D} 138 \mathrm{~N} / \mathrm{D} 138 \mathrm{~N}} \mathrm{BMDMs}$ or pharmacologically by Necrostatin-1s did not inhibit the LPS-induced release of IL-1 $\beta$ and IL-1 $\alpha$ from A20-deficient macrophages (Fig. 6a, b), suggesting that RIPK1 kinase activity is dispensable for the production of these cytokines under in vitro stimulation conditions. Taken together, these results suggested that MLKL-dependent necroptosis contributes to both the inflammasome-dependent release of IL-1 $\beta$ and the inflammasome-independent production of IL-1 $\alpha$ by A20-deficient macrophages.

Previous work suggested that RIPK3 regulates LPS-induced IL-1 $\beta$ release by A20-deficient macrophages independently of cell death ${ }^{39}$. However, one caveat of assessing cell death using biochemical assays such as LDH release is that these methodologies are not sensitive enough to detect the death of a small fraction of the cells. Based on our results above, we reasoned that IL-1 $\beta$ could be released by a small fraction of dying A20-deficient macrophages. To test this hypothesis, we employed newly a developed methodology allowing the dynamic real-time imaging of cell death and IL-1 $\beta$ release simultaneously at the single cell level ${ }^{49,50}$. BMDMs from wild type, $\mathrm{A} 20^{\mathrm{MYC}-\mathrm{KO}}$ and A20 ${ }^{\mathrm{MYC}-\mathrm{KO}}$ Ripk ${ }^{-1-}$ mice seeded on a microwell device were stimulated with LPS and cell death and IL-1 $\beta$ secretion were measured at single-cell resolution using total internal reflection fluorescent microscopy. As shown in Fig. 6c, a considerable fraction of dying A20-deficient BMDMs secreted IL$1 \beta$, while surviving cells did not secrete the cytokine. Moreover, RIPK3 deficiency strongly suppressed both cell death and IL-1 $\beta$ release in LPS-stimulated A20 knockout macrophages (Fig. $6 \mathrm{c}, \mathrm{d}$ ). We then used a modified protocol allowing the concurrent detection of both IL-1 $\alpha$ and IL-1 $\beta$ release as well as cell death and found that in the majority of the cases both cytokines were secreted simultaneously from the dying cells (Fig. 6e). Therefore, the LPS-induced release of IL-1 $\beta$ but also IL-1 $\alpha$ from A20-deficient macrophages requires RIPK3-dependent cell death.

\section{ZnF7 is essential for A20-mediated inhibition of inflammation}

Knock-in mice expressing A20 with mutations in its catalytic OTU domain or its ZnF4 domain did not develop spontaneous inflammatory pathology $28,30,31$, suggesting that other domains are essential for inhibition of inflammation in vivo. The $\mathrm{ZnF7}$ of $\mathrm{A} 20$ has been shown to play an important role in 
both inhibition of NF- $\mathrm{kB}$ activation and cell death downstream of TNFR1 in cell line reconstitution experiments $^{32,35,37}$, but its role in the regulation of inflammation in vivo has not been studied. We therefore generated knock-in mice expressing A20 with a mutated ZnF7 domain by mutating cysteine residues 764 and 767 to alanines (C764A, C767A, hereafter referred to as $\mathrm{mZnF7}$ ) (Supplementary Fig. 5a). These mutations abrogate the ability of A20 to bind to polyubiquitin chains $^{32}$. Homozygous $A 20^{\mathrm{mZnF} 7 / \mathrm{MZnF7}}$ mice were born at Mendelian ratio and did not show the early postnatal lethality observed in $A 2 \mathrm{O}^{-/-}$mice, but had reduced body weight and developed symmetric swelling in their ankles, wrists and toes that became obvious from the age of 5 weeks (Fig. $7 \mathrm{a}, \mathrm{C}$ ). $A 20^{\mathrm{mZnF} / \mathrm{mZnF7}}$ mice showed splenomegaly similarly to $\mathrm{A} 20^{\mathrm{MYC}-\mathrm{KO}}$ mice (Fig. $7 \mathrm{~b}$ ). Ex vivo microcomputed tomography (microCT) analysis of hind and forepaws revealed erosions and new bone formation in the joints of $A 20^{\mathrm{mZnF} 7 / \mathrm{mZnF7}}$ mice indicative of inflammatory bone remodelling, similarly to the $\mathrm{A} 20^{\mathrm{MYC}-\mathrm{KO}}$ animals (Fig. $7 \mathrm{~d}$ and Supplementary Fig. $5 \mathrm{~b}$ ). In addition, $A 20^{\mathrm{mZnF} 7 / \mathrm{mZnF} 7}$ mice showed severe erosions of all distal phalanges and nails (Supplementary Fig. 5c), suggestive of a dactylitislike phenotype characterized by the formation of a pannus tissue and destruction of the nail bed and the underlying distal phalanx. Histological analysis of the ankle joints revealed clear signs of inflammatory arthritis manifesting a persisting synovitis evolving into pannus and leading to apparent proteoglycan degradation in articular cartilage, as this is exhibited through the loss of toluidine blue staining, as well as activation of osteo-resorptive processes in the adjacent bone (Figure $7 \mathrm{e}-\mathrm{h}$ ). Therefore, $A 20^{\mathrm{mZnF} 7 / \mathrm{mZnF7}}$ mice developed progressive inflammatory joint pathology although the overall severity of the arthritis observed in the ankles of these animals was generally milder compared to age-matched $\mathrm{A} 20^{\mathrm{MYC}-\mathrm{KO}}$ mice. Increased neutrophil infiltration was observed in the joints of $\mathrm{A} 20^{\mathrm{MYC}-\mathrm{KO}}$ compared to $A 20^{\mathrm{mZnF} / \mathrm{mZnF7}}$ mice (Supplementary Fig. 6), which could account for the accelerated development and progression of arthritis in the A20 ${ }^{\mathrm{MYC}-\mathrm{KO}}$ mice ${ }^{51}$. In addition, $A 20^{\mathrm{mZnF} / \mathrm{mZnF7}}$ mice developed severe inflammation in their distal toe joints, which was never observed in $\mathrm{A} 2 \mathrm{O}^{\mathrm{MYC}-\mathrm{KO}}$ animals, suggesting that non-myeloid cell dependent mechanisms account for this pathology. We then assessed whether MyD88-dependent signalling drives arthritis development also in $A 20^{\mathrm{mZnF} / \mathrm{mZnF} 7}$ mice, as is seen in $\mathrm{A} 20^{\mathrm{MYC}-\mathrm{KO}}$ animals. As shown in Supplementary Fig. 7, MyD88 deficiency partly restored the reduced body weight and splenomegaly but fully prevented the development of arthritis and dactylitis in $A 20^{\mathrm{mZnF} / \mathrm{mZnF7}}$ mice, showing that MyD88-dependent mechanisms drive arthritis development in the absence of ZnF7-dependent A20 functions similarly to $\mathrm{A} 20^{\mathrm{MYC}-\mathrm{KO}}$ mice. Taken together, these results showed that the $\mathrm{ZnF} 7$ has a critical indispensable in vivo function for A20-mediated repression of joint inflammation.

LPS stimulation induced the release of processed IL-1 $\beta$ as well as IL- $1 \alpha$ in $A 20^{\mathrm{mZnF7} / \mathrm{mZnF7}}$ BMDMs, although at lower levels compared to A20 knockout cells (Fig. 8a, b). The ZnF7 has been implicated in facilitating the recruitment of A20 to the TNFR1 signalling complex by binding linear ubiquitin chains $^{37}$. Indeed, FLAG-TNF immunoprecipitation experiments showed that ZnF7 mutation almost completely prevented the recruitment of A20 to complex I (Figure 8c). Immunoblot analysis of FLAG-TNF immunoprecipitates with anti-RIPK1 antibodies revealed efficient recruitment and ubiquitination of RIPK1 in complex I in $A 20^{\mathrm{mZnF7} / \mathrm{mZnF7}}$ as well as in A20-deficient BMDMs (Figure 8d). However, immunoblot with antibodies specifically recognizing M1-linked ubiquitin chains revealed a strong reduction of linear ubiquitin chains within complex I in both $A 20^{\mathrm{mZnF} / \mathrm{mZnF7}}$ as well as in $\mathrm{A} 20$ deficient BMDMs compared to wild-type BMDMs, consistent with the concept that A20 binding stabilises linear ubiquitin chains likely by preventing their degradation by other DUBs ${ }^{37}$. Therefore, $\mathrm{ZnF7}$ mutation abolishes the recruitment of $\mathrm{A} 20$ and reduces the amount of linear ubiquitin chains in the TNFR1 signalling complex.

\section{Discussion}

Multiple studies have established A20 as a key regulator of inflammation in animal models ${ }^{3,8-10,12,14}$, $15,17-27,38,39$ and in human patients ${ }^{6-13}$, but the molecular mechanisms and the functional domains of A20 that are critical to prevent inflammation in vivo have remained elusive. Our results showing that mice expressing A20 with mutated ZnF7 spontaneously develop inflammatory joint pathology identify ZnF7-dependent binding to linear ubiquitin chains as a critical function of A20 that is indispensable for inhibition of inflammation in vivo. However, the spontaneous inflammatory pathology developing in $A 20^{\mathrm{mZnF} / \mathrm{mZnF7}}$ mice is mild compared to the severe inflammation causing 
early postnatal lethality in $A 20$ knockout animals ${ }^{14}$, suggesting that other domains of A20 also critically contribute to its in vivo anti-inflammatory function. Generation of knock-in mice with combined domain mutations, which for time limitations could not be included in this study, will be important to dissect redundancies between the different functional domains of A20.

Inhibition of NF- $\kappa B$ activation is considered a key anti-inflammatory function of $A 20(2,27)$, while its role in preventing cell death has received little attention as a possible mechanism suppressing inflammation. Surprisingly, inhibition of IKK/NF-кB signalling in A20-deficient myeloid cells was not sufficient to prevent arthritis development. Instead, our results identified regulation of necroptosis as a critical anti-inflammatory function of A20 that is important to prevent the development of arthritis in mice. RIPK1/RIPK3/MLKL-dependent necroptosis was required for inflammasome activation, IL-1 $\beta$ production and arthritis development in $\mathrm{A} 20^{\mathrm{MYC}-\mathrm{KO}}$ mice, providing evidence that $\mathrm{A} 20$ suppresses inflammasome activation in vivo indirectly by inhibiting necroptosis. Plasma membrane permeabilization by MLKL likely triggers NLRP3 inflammasome activation by inducing potassium efflux ${ }^{52}$. While an earlier study suggested that LPS induces IL-1 $\beta$ secretion in A20 knockout macrophages in the absence of cell death ${ }^{39}$, our single cell imaging experiments demonstrated that $\mathrm{IL}-1 \beta$, as well as IL-1 $\alpha$, are secreted exclusively by a small number of dying A20-deficient macrophages. ASC deficiency could prevent IL-1 $\beta$ but not IL-1 $\alpha$ release from A20-knockout macrophages, providing additional evidence that necroptosis and not pyroptosis drives the death of these cells in response to LPS stimulation. Our results also question earlier reports arguing that RIPK3 regulates inflammasome activation and IL-1 $\beta$ release independently of cell death ${ }^{53,54}$. Reevaluation of these results using dynamic real-time imaging of cell death and IL-1 $\beta$ release at a single cell level will be required to clarify whether RIPK3-dependent IL-1 $\beta$ secretion occurs independently of cell death. MLKL deficiency was as efficient as RIPK3 knockout in preventing IL-1 $\beta$ release and arthritis in vivo in $\mathrm{A} 2 \mathrm{O}^{\mathrm{MYC}-\mathrm{KO}}$ mice, but only partly reduced LPS-induced IL-1 $\beta$ production from A20 knockout macrophages in vitro. Z-VAD-fmk could prevent IL- $1 \alpha$ and IL-1 $\beta$ release in A20/MLKL double-deficient BMDMs, suggesting that caspase-dependent cell death drives the release of these cytokines in the absence of MLKL. It is likely that secondary necrosis of apoptotic A20/MLKL double-deficient macrophages is responsible for the release of IL-1 $\beta$ and IL-1 $\alpha$ in vitro, while these cells may be rapidly cleared by phagocytes in vivo thus preventing secondary necrosis. Our work also identified synovial fibroblasts as the key effector cells that respond to IL-1 family cytokines secreted by the A20-deficient myeloid cells within the joints, further driving the inflammatory response leading to the development of arthritis (Supplementary Fig. 8).

RIPK3 deficiency or inhibition of RIPK1 kinase activity considerably delayed the early postnatal lethality of $A 20^{-1-}$ mice ${ }^{15,16}$, however MLKL knockout did not show any protective effect ${ }^{16}$. Therefore, A20 deficiency in non-myeloid cells causes inflammation by inducing necroptosis-independent functions of RIPK1 and RIPK3. RIPK1 and RIPK3 can induce apoptosis but have also been reported to exert cell death-independent proinflammatory functions ${ }^{45}$. Apoptosis of A20-deficient epithelial or stromal cells likely contributes to systemic inflammation in $\mathrm{A2O}^{-1-}$ as shown in conditional A20 knockout animals ${ }^{18,22,25}$. Unequivocal assessment of the role of cell death in the pathology caused by systemic A20 deficiency will require the combined inhibition of both FADD/Caspase-8mediated apoptosis and MLKL-dependent necroptosis.

The ligands and receptors triggering necroptosis of A20-deficient macrophages in vivo are not known at present. Although TNFR1 deficiency did not prevent arthritis in A20 ${ }^{\mathrm{MYC}-\mathrm{KO}}$ animals ${ }^{23}$, we cannot exclude that TNFR1 could contribute to necroptosis of A20-deficient macrophages in vivo in a redundant fashion with other upstream pathways, such as TRIF-dependent TLR3/TLR4 signalling or type I and type II interferon receptor signalling. TLR4 could act in a dual fashion, providing a MyD88-dependent 'priming' signal inducing the transcriptional upregulation of inflammasome components as well as IL-1 $\alpha$ and IL-1 $\beta$ and in parallel activating necroptosis directly via TRIF or indirectly by autocrine TNF production. 
Taken together, our findings demonstrate that regulation of macrophage necroptosis is a key in vivo anti-inflammatory function of A20. In addition, our results identify macrophage necroptosis as a potent in vivo trigger of inflammasome activation and arthritis development, suggesting that necroptosis and perhaps other mechanisms causing plasma membrane permeabilization of 'primed' myeloid cells could contribute to inflammasome-associated pathologies. Therefore, inhibitors of necroptosis, as for example RIPK1 inhibitors, could prove effective for the treatment of chronic inflammatory diseases associated with impaired A20 function as well as inflammasome activation.

\section{References}

1. Lork, M., Verhelst, K. \& Beyaert, R. CYLD, A20 and OTULIN deubiquitinases in NF-kappaB signaling and cell death: so similar, yet so different. Cell Death Differ 24, 1172-1183 (2017).

2. Ma, A. \& Malynn, B.A. A20: linking a complex regulator of ubiquitylation to immunity and human disease. Nat Rev Immunol 12, 774-785 (2012).

3. Das, T., Chen, Z., Hendriks, R.W. \& Kool, M. A20/Tumor Necrosis Factor alpha-Induced Protein 3 in Immune Cells Controls Development of Autoinflammation and Autoimmunity: Lessons from Mouse Models. Front Immunol 9, 104 (2018).

4. Vereecke, L., Beyaert, R. \& van Loo, G. Genetic relationships between A20/TNFAIP3, chronic inflammation and autoimmune disease. Biochem Soc Trans 39, 1086-1091 (2011).

5. Novak, U. et al. The NF-\{kappa\}B negative regulator TNFAIP3 (A20) is inactivated by somatic mutations and genomic deletions in marginal zone lymphomas. Blood 113, 4918-4921 (2009).

6. Kato, M. et al. Frequent inactivation of A20 in B-cell lymphomas. Nature 459, 712-716 (2009).

7. Schmitz, R. et al. TNFAIP3 (A20) is a tumor suppressor gene in Hodgkin lymphoma and primary mediastinal B cell lymphoma. J Exp Med 206, 981-989 (2009).

8. Compagno, M. et al. Mutations of multiple genes cause deregulation of NF-kappaB in diffuse large Bcell lymphoma. Nature 459, 717-721 (2009).

9. Aeschlimann, F.A. et al. A20 haploinsufficiency (HA20): clinical phenotypes and disease course of patients with a newly recognised NF-kB-mediated autoinflammatory disease. Ann Rheum Dis 77, 728-735 (2018).

10. Duncan, C.J.A. et al. Early-onset autoimmune disease due to a heterozygous loss-of-function mutation in TNFAIP3 (A20). Ann Rheum Dis 77, 783-786 (2018).

11. Kadowaki, T. et al. Haploinsufficiency of A20 causes autoinflammatory and autoimmune disorders. $J$ Allergy Clin Immunol 141, 1485-1488 e1411 (2018).

12. Takagi, M. et al. Haploinsufficiency of TNFAIP3 (A20) by germline mutation is involved in autoimmune lymphoproliferative syndrome. J Allergy Clin Immunol 139, 1914-1922 (2017).

13. Zhou, Q. et al. Loss-of-function mutations in TNFAIP3 leading to A20 haploinsufficiency cause an early-onset autoinflammatory disease. Nat Genet 48, 67-73 (2016).

14. Lee, E.G. et al. Failure to regulate TNF-induced NF-kappaB and cell death responses in A20-deficient mice. Science 289, 2350-2354 (2000).

15. Onizawa, M. et al. The ubiquitin-modifying enzyme A20 restricts ubiquitination of the kinase RIPK3 and protects cells from necroptosis. Nat Immunol 16, 618-627 (2015).

16. Newton, K. et al. RIPK3 deficiency or catalytically inactive RIPK1 provides greater benefit than MLKL deficiency in mouse models of inflammation and tissue injury. Cell Death Differ 23, 1565-1576 (2016).

17. Tavares, R.M. et al. The ubiquitin modifying enzyme A20 restricts B cell survival and prevents autoimmunity. Immunity 33, 181-191 (2010).

18. Vereecke, L. et al. Enterocyte-specific A20 deficiency sensitizes to tumor necrosis factor-induced toxicity and experimental colitis. J Exp Med 207, 1513-1523 (2010).

19. Chu, Y. et al. B cells lacking the tumor suppressor TNFAIP3/A20 display impaired differentiation and hyperactivation and cause inflammation and autoimmunity in aged mice. Blood 117, 2227-2236 (2011). 
20. Hammer, G.E. et al. Expression of A20 by dendritic cells preserves immune homeostasis and prevents colitis and spondyloarthritis. Nat Immunol 12, 1184-1193 (2011).

21. Kool, M. et al. The ubiquitin-editing protein A20 prevents dendritic cell activation, recognition of apoptotic cells, and systemic autoimmunity. Immunity 35, 82-96 (2011).

22. Lippens, S. et al. Keratinocyte-specific ablation of the NF-kappaB regulatory protein A20 (TNFAIP3) reveals a role in the control of epidermal homeostasis. Cell Death Differ 18, 1845-1853 (2011).

23. Matmati, M. et al. A20 (TNFAIP3) deficiency in myeloid cells triggers erosive polyarthritis resembling rheumatoid arthritis. Nat Genet 43, 908-912 (2011).

24. Heger, K. et al. A20-deficient mast cells exacerbate inflammatory responses in vivo. PLoS Biol 12, e1001762 (2014).

25. Catrysse, L. et al. A20 prevents chronic liver inflammation and cancer by protecting hepatocytes from death. Cell Death Dis 7, e2250 (2016).

26. Voet, S. et al. A20 critically controls microglia activation and inhibits inflammasome-dependent neuroinflammation. Nat Commun 9, 2036 (2018).

27. Catrysse, L., Vereecke, L., Beyaert, R. \& van Loo, G. A20 in inflammation and autoimmunity. Trends Immunol 35, 22-31 (2014).

28. Wertz, I.E. et al. Phosphorylation and linear ubiquitin direct A20 inhibition of inflammation. Nature 528, 370-375 (2015).

29. Wertz, I.E. et al. De-ubiquitination and ubiquitin ligase domains of A20 downregulate NF-kappaB signalling. Nature 430, 694-699 (2004).

30. Lu, T.T. et al. Dimerization and ubiquitin mediated recruitment of A20, a complex deubiquitinating enzyme. Immunity 38, 896-905 (2013).

31. De, A., Dainichi, T., Rathinam, C.V. \& Ghosh, S. The deubiquitinase activity of A20 is dispensable for NF-kappaB signaling. EMBO Rep 15, 775-783 (2014).

32. Skaug, B. et al. Direct, noncatalytic mechanism of IKK inhibition by A20. Mol Cell 44, 559-571 (2011).

33. Tokunaga, F. et al. Specific recognition of linear polyubiquitin by A20 zinc finger 7 is involved in NFkappaB regulation. EMBO J 31, 3856-3870 (2012).

34. Verhelst, K. et al. A20 inhibits LUBAC-mediated NF-kappaB activation by binding linear polyubiquitin chains via its zinc finger 7. EMBO J 31, 3845-3855 (2012).

35. Yamaguchi, N. \& Yamaguchi, N. The seventh zinc finger motif of A20 is required for the suppression of TNF-alpha-induced apoptosis. FEBS Lett 589, 1369-1375 (2015).

36. Bosanac, I. et al. Ubiquitin binding to A20 ZnF4 is required for modulation of NF-kappaB signaling. Mol Cell 40, 548-557 (2010).

37. Draber, P. et al. LUBAC-Recruited CYLD and A20 Regulate Gene Activation and Cell Death by Exerting Opposing Effects on Linear Ubiquitin in Signaling Complexes. Cell Rep 13, 2258-2272 (2015).

38. Vande Walle, L. et al. Negative regulation of the NLRP3 inflammasome by A20 protects against arthritis. Nature 512, 69-73 (2014).

39. Duong, B.H. et al. A20 restricts ubiquitination of pro-interleukin-1beta protein complexes and suppresses NLRP3 inflammasome activity. Immunity 42, 55-67 (2015).

40. Lamkanfi, M. \& Dixit, V.M. Mechanisms and functions of inflammasomes. Cell 157, 1013-1022 (2014).

41. Gais, P. et al. Cutting edge: Divergent cell-specific functions of MyD88 for inflammatory responses and organ injury in septic peritonitis. J Immunol 188, 5833-5837 (2012).

42. McInnes, I.B. \& Schett, G. The pathogenesis of rheumatoid arthritis. N Engl J Med 365, 2205-2219 (2011).

43. Smolen, J.S., Aletaha, D. \& McInnes, I.B. Rheumatoid arthritis. Lancet 388, 2023-2038 (2016).

44. Armaka, M. et al. Mesenchymal cell targeting by TNF as a common pathogenic principle in chronic inflammatory joint and intestinal diseases. J Exp Med 205, 331-337 (2008).

45. Moriwaki, K. \& Chan, F.K. The Inflammatory Signal Adaptor RIPK3: Functions Beyond Necroptosis. Int Rev Cell Mol Biol 328, 253-275 (2017). 
46. Pasparakis, M. \& Vandenabeele, P. Necroptosis and its role in inflammation. Nature 517, 311-320 (2015).

47. Polykratis, A. et al. Cutting edge: RIPK1 Kinase inactive mice are viable and protected from TNFinduced necroptosis in vivo. J Immunol 193, 1539-1543 (2014).

48. Garlanda, C., Dinarello, C.A. \& Mantovani, A. The interleukin-1 family: back to the future. Immunity 39, 1003-1018 (2013).

49. Liu, T. et al. Single-cell imaging of caspase-1 dynamics reveals an all-or-none inflammasome signaling response. Cell Rep 8, 974-982 (2014).

50. Shirasaki, Y. et al. Real-time single-cell imaging of protein secretion. Sci Rep 4, 4736 (2014).

51. Wright, H.L., Moots, R.J. \& Edwards, S.W. The multifactorial role of neutrophils in rheumatoid arthritis. Nat Rev Rheumatol 10, 593-601 (2014).

52. Conos, S.A. et al. Active MLKL triggers the NLRP3 inflammasome in a cell-intrinsic manner. Proc Natl Acad Sci U S A 114, E961-E969 (2017).

53. Kang, T.B., Yang, S.H., Toth, B., Kovalenko, A. \& Wallach, D. Caspase-8 blocks kinase RIPK3-mediated activation of the NLRP3 inflammasome. Immunity 38, 27-40 (2013).

54. Vince, J.E. et al. Inhibitor of apoptosis proteins limit RIP3 kinase-dependent interleukin-1 activation. Immunity 36, 215-227 (2012).

\section{Author contributions}

A.P., G.vL., M.A. and M.P. conceived the study and designed the experiments. A.P., A.M., O.E., Y.S., M.Y., Y.Y. and M.A. performed and analysed experiments. B.H. provided mice. Y.Y., S.U., M.M., G.K., M.A., G.vL. and MP supervised the experiments. A.P., M.A., G.vL and MP interpreted data and wrote the paper.

\section{Acknowledgments}

We are grateful to J. Buchholz, C. Uthoff-Hachenberg, E. Mahlberg, B. Kühnel, E. Stade, T. Liu, B. Yao, K. Igarashi, M. Sze, L. Bellen, S. Lalos, P. Athanasakis and A. Kateveni for excellent technical assistance, and T. Hochepied for help with the generation of $A 20^{\mathrm{mZnF} 7 / \mathrm{mZnF7}}$ mice. We also thank $\mathrm{D}$. Hackam for providing TIr $4^{\mathrm{FL} / \mathrm{FL}}$ mice, V. Dixit and Genentech for providing Ripk $3^{-1-}$ mice, G. Vassilopoulos for providing access to microscope facilities, and the InfrafrontierGR infrastructure (NSRF 2007-2013 and NSRF 2014-2020) for providing mouse hosting and mCT facilities. This work was supported by funding from the European Research Council (ERC AdG 323040 and 787826 to MP; AdG 340217 to GK), the Greek GSRT project INNATE FIBLROBLAST to GK (ERC06, cofinanced by the ESF and NSRF 2007-2013), the European Commission (FP7 grant 'Masterswitch' 223404 to MP and GK), by JSPS KAKENHI Grant Number JP16H06385 to M.M, JP26110005 to Y.Y., JP15H01366, JP17H05496 to YS, JST, PRESTO Grant Number JP17940748 to Y.S., and the Japan Agency for Medical Research and Development (JP17gm0610004 and JP17gm5010001) to M.M. Research in the G.v.L. lab is supported by research grants from the FWO, the "Geneeskundige Stichting Koningin Elisabeth" (GSKE), the CBC Banque Prize, the Charcot Foundation, the "Belgian Foundation against Cancer", and "Kom op tegen Kanker". A. Martens is supported by a grant from the "Concerted Research Actions" (GOA) of the Ghent University. Research in the M.A. lab is supported by a startup grant from the Stavros Niarchos Foundation donation to BSRC "Al. Fleming". MA and GK also acknowledge support of this work by project MIS 5002562 funded by NSRF 2014-2020, co-financed by Greece and the European Union (ERDF). R.O.E. was supported by a postdoctoral fellowship from the Alexander von Humboldt foundation.

\section{Declaration of competing interests}

The authors declare the following competing interests: M.P. received consulting and speaker fees from Genentech, GSK and Boehringer. 


\section{Figure Legends.}

Figure 1. Myeloid cell specific ASC knockout inhibits arthritis development in A20 ${ }^{\text {MYC-KO }}$ mice. (a) Graphs depicting spleen weight of mice with the indicated genotypes. (b) Graph showing serum IL-1 $\beta$ levels in mice with the indicated genotypes ( $n=10$ mice per genotype). (c) Representative macroscopic and histological images of the ankle joints of mice with the indicated genotypes. Bone erosion was detected by tartrate-resistant acid phosphatase (TRAP) staining of osteoclast activity and cartilage destruction was assessed by proteoglycan staining with toluidine blue. (scale bar: 500 $\mu \mathrm{m})$. (d-h) Graphs depicting weekly clinical scores (d), the average thickness of rear paws at the ankle area (e), as well as histological scores for inflammation (f), bone erosion (g), and cartilage destruction ( $\mathrm{h}$ ) in mice with the indicated genotypes. Mice were analysed at the age of $23-27$ weeks (wt, n=16; $A 20^{\mathrm{MYC}-\mathrm{KO}}, \mathrm{n}=35 ; \mathrm{A} 20^{\mathrm{MYC}-\mathrm{KO}} \mathrm{Asc}^{\mathrm{MYC}-\mathrm{KO}}, \mathrm{n}=25$ mice for $\mathrm{a}, \mathrm{c}-\mathrm{h}$ ). i) Graphs depicting the levels of released IL-1 $1 \beta$ and IL-1 $\alpha$ in the supernatants of BMDMs stimulated for 24 hours with 1 $\mu \mathrm{g} / \mathrm{ml}$ of ultrapure LPS. Graphs show pooled results of three independent experiments, performed at different times with one independent isolation of macrophages from the indicated genotypes per experiment $(n=3$ experiments on independent isolations of BMDMs). In all graphs mean \pm SEM is shown for each group of mice. ${ }^{*},{ }^{* *}$ and ${ }^{* * *}$ represent $p<0.05, p<0.01$ and $p<0.001$ respectively (non-parametric Mann-Whitney test between indicated genotypes for a, b, e-h; two-way ANOVA post-Bonferroni test for d; one-way ANOVA post-Bonferroni test for i). All statistical tests are twotailed. Raw data are provided in Supplementary Table 3.

Figure 2. MyD88-dependent signalling in both myeloid and non-myeloid cells is required for spontaneous arthritis development in $\mathbf{A 2 0}{ }^{\text {MYC-Ko }}$ mice. (a) Graph depicting the spleen weight of mice with the indicated genotypes. (b) Graph showing serum IL-1 $\beta$ levels in mice with the indicated genotypes ( $n=10$ mice per genotype). (c) Representative macroscopic and histological images of the ankle joints of mice with the indicated genotypes (scale bar: $500 \mu \mathrm{m})$. (d-h) Graphs depicting weekly clinical scores (d), the average thickness of rear paws at the ankle area (e), as well as histological scores for inflammation (f), bone erosion (g), and cartilage destruction (h) in mice with the indicated genotypes. Mice were analysed at the age of $23-27$ weeks, wt and $\mathrm{A} 20^{\mathrm{MYC}-\mathrm{KO}}$ data are same as in figure 1 and are included for comparison (wt, $n=16 ; A 20^{\mathrm{MYC}-\mathrm{KO}}, \mathrm{n}=35$; $\mathrm{A} 20^{\mathrm{MYC}-\mathrm{KO}}$ MyD88 $^{\mathrm{MYC}-\mathrm{KO}}, \mathrm{n=15}$; $\mathrm{A}^{\mathrm{MYC} 0^{\mathrm{MYO}}} \mathrm{MyD}^{\mathrm{MYC}} \mathrm{n=17}$ mice for $\left.\mathrm{a}, \mathrm{c}-\mathrm{h}\right)$. (i) Graphs depicting the levels of released IL-1 $\beta$ and IL-1 $\alpha$ in the supernatants of BMDMs stimulated for 24 hours with $1 \mu \mathrm{g} / \mathrm{ml}$ of ultrapure LPS. Graphs show pooled results of three independent experiments, performed at different times with one independent isolation of macrophages from the indicated genotypes per experiment ( $n=3$ experiments on independent isolations of BMDMs). (j) Graphs depicting mRNA expression levels of IL-1 $\beta$ and IL- $1 \alpha$ in macrophages stimulated with $20 \mathrm{ng} / \mathrm{ml}$ of LPS. The graph shows the result of one experiment performed with three independent isolations of BMDMs with the indicated genotypes ( $n=3$ independent isolations of BMDMs per genotype). In all graphs mean \pm SEM is shown for each group of mice. ${ }^{*},{ }^{* *}$ and ${ }^{* * *}$ represent $p<0.05, p<0.01$ and $p<0.001$ respectively (non-parametric Mann-Whitney test between indicated genotypes for $a, b$, e-h; two-way ANOVA post-Bonferroni test for $\mathrm{d}$, j; one-way ANOVA post-Bonferroni test for i). All statistical tests are two-tailed. Raw data are provided in Supplementary Table 3.

Figure 3. Synovial fibroblast-specific MyD88-dependent signalling contributes to spontaneous development of arthritis in A20 ${ }^{\mathrm{MYC}-\mathrm{KO}}$ mice. (a) Representative macroscopic and histological images of the ankle joints of mice with the indicated genotypes (scale bar: $500 \mu \mathrm{m})$. (b-f) Graphs depicting weekly clinical scores (b), average thickness of rear paws at the ankle area (c), as well as histological scores for inflammation (d), bone erosion (e), and cartilage destruction (f) in mice with the indicated genotypes. Mice were analysed 25 weeks after bone marrow transfer. Dots in the graphs indicate individual mice (wt to wt, $n=7$; wt to MyD88 ${ }^{\text {SF-KO }} n=8 ; A 20^{\text {MYC-KO }}$ to wt, $n=11$, $\mathrm{A} 20^{\mathrm{MYC}-\mathrm{KO}}$ to MyD88 ${ }^{\mathrm{SF}-\mathrm{KO}}, \mathrm{n}=10$ mice for $\left.\mathrm{a}-\mathrm{f}\right)$. In all graphs mean \pm SEM is shown for each group of mice. ${ }^{*}$ and ${ }^{* *}$ represent $p<0.05$ and $p<0.01$ respectively (non-parametric Mann-Whitney test between indicated genotypes for c-f; two-way ANOVA post-Bonferroni test for b). All statistical tests are two-tailed. Raw data are provided in Supplementary Table 3. 
Figure 4. IKK2 deficiency in myeloid cells does not inhibit the development of arthritis in A20 ${ }^{\text {MYC-KO }}$ mice. (a) Graphs depicting spleen weight of mice with the indicated genotypes. (b) Graph showing serum IL-1 $\beta$ levels in mice with the indicated genotypes ( $n=10$ mice per genotype). (c) Representative macroscopic and histological images of the ankle joints of mice with the indicated genotypes (scale bar: $500 \mu \mathrm{m}$ ). (d-h) Graphs depicting weekly clinical scores (d), the average thickness of rear paws at the ankle area (e), as well as histological scores for inflammation (f), bone erosion ( $\mathrm{g}$ ), and cartilage destruction (h) in mice with the indicated genotypes. Mice were analysed at the age of $23-27$ weeks, wt and $\mathrm{A} 20^{\mathrm{MYC}-\mathrm{KO}}$ data are same as in figure 1 and are included for comparison (wt, $\mathrm{n}=16 ; \mathrm{A} 20^{\mathrm{MYC}-\mathrm{KO}}, \mathrm{n}=35 ; \mathrm{A} 20^{\mathrm{MYC}-\mathrm{KO}} \mathrm{IKK} 2^{\mathrm{MYC}-\mathrm{KO}}, \mathrm{n}=12$ mice for $\mathrm{a}, \mathrm{c}-\mathrm{h}$ ). In all graphs mean \pm SEM is shown for each group of mice. ${ }^{* * *}$ represents $p<0.001$ (non-parametric MannWhitney test between indicated genotypes for a, b, e-h; two-way ANOVA post-Bonferroni test for d). All statistical tests are two-tailed. Raw data are provided in Supplementary Table 3.

Figure 5. Necroptosis contributes to spontaneous development of arthritis in $\mathrm{A}^{20^{\mathrm{MYC}-\mathrm{KO}}}$ mice. (a) Representative macroscopic and histological images of the ankle joints of mice with the indicated genotypes (scale bar: $500 \mu \mathrm{m}$ ). (b-f) Graphs depicting weekly clinical scores (b), the average thickness of rear paws at the ankle area (c), as well as histological scores for inflammation (d), bone erosion (e), and cartilage destruction (f) in mice with the indicated genotypes. Mice were analysed at the age of $23-27$ weeks, wt and $\mathrm{A} 20^{\mathrm{MYC}-\mathrm{KO}}$ data are same as in figure 1 and are

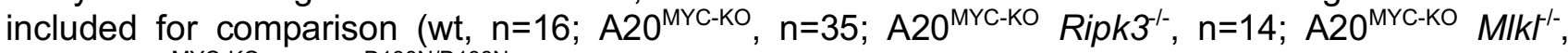
$\mathrm{n}=12 ; \mathrm{A} 20^{\mathrm{MYC}-\mathrm{KO}} \operatorname{Ripk} 1^{\mathrm{D} 138 \mathrm{~N} / \mathrm{D} 138 \mathrm{~N}}, \mathrm{n}=14$ mice for $\left.\mathrm{a}-\mathrm{f}\right)$. (g) Graph showing serum IL-1 $\beta$ levels in mice with the indicated genotypes ( $n=10$ mice per genotype). (h) Graphs indicating the number of white blood cells (WBC), lymphocytes (LY), monocytes (MO), and neutrophils (NE) in peripheral blood from mice with the indicated genotypes. Wild type and $\mathrm{A} 20^{\mathrm{MYC}-\mathrm{KO}}$ mice are the same shown in Supplementary Fig. 1 and are included for comparison (wt, n=54; 220 $^{\mathrm{MYC}-\mathrm{KO}}, \mathrm{n}=33 ; \mathrm{A} 20^{\mathrm{MYC}-\mathrm{KO}}$ Ripk3I- $\mathrm{n}=18 ; \mathrm{A} 20^{\mathrm{MYC}-\mathrm{KO}} \mathrm{MlkI}^{--}, \mathrm{n}=22 ; \mathrm{A} 20^{\mathrm{MYC}-\mathrm{KO}} \operatorname{Ripk}^{\mathrm{D} 138 \mathrm{~N} / \mathrm{D} 138 \mathrm{~N}}, \mathrm{n}=26$ mice). (i) Levels of the indicated cytokines and chemokines in the serum of mice with the indicated genotypes. $(n=10$ mice per genotype; for IL-18, $\mathrm{n}=9$ mice for $\mathrm{A} 20^{\mathrm{MYC}-\mathrm{KO}}$ Ripk $3^{-1-}$ ). Dots in the graphs indicate individual mice. In all graphs mean \pm SEM is also shown for each group of mice. ${ }^{*},{ }^{* *}$ and ${ }^{* * *}$ represent $p<0.05, p<0.01$ and $p<0.001$ respectively (non-parametric Mann-Whitney test between indicated genotypes for c-g; two-way ANOVA post-Bonferroni test for b). All statistical tests are two-tailed. Raw data are provided in Supplementary Table 3.

Figure 6. RIPK3-dependent necroptosis and apoptosis trigger LPS-induced IL-1 $\beta$ and IL-1 $\alpha$ secretion from A20-deficient macrophages. (a) BMDMs with the indicated genotypes were stimulated with $1 \mu \mathrm{g} / \mathrm{ml}$ LPS and the indicated proteins were analysed in cell culture supernatants or cell lysates by immunobloting. Data from one representative out of three independent experiments shown. (b) Graphs depicting the levels of released IL-1 $\beta$ and IL-1 $\alpha$ in supernatants of BMDMs from mice with the indicated genotypes stimulated for 24 hours with $1 \mu \mathrm{g} / \mathrm{ml}$ of ultrapure LPS in combination with Necrostatin-1s (N) or Z-VAD-fmk (Z) as indicated. Graphs show pooled results of three independent experiments, performed at different times with one independent isolation of macrophages from the indicated genotypes per experiment $(n=3$ experiments on independent isolations of BMDMs). In all graphs mean \pm SEM is also shown for each group of mice. *, ** and *** represent $p<0.05, p<0.01$ and $p<0.001$ respectively (two-way ANOVA post-Bonferroni test between indicated genotypes). (c) Dot-plot representation of the signal intensity of IL-1 $\beta$ secreted from single cells, classified according to cell viability, analysed in BMDMs with the indicated genotypes stimulated with $2 \mu \mathrm{g} / \mathrm{ml}$ of crude LPS for 10 hours. ${ }^{*},{ }^{* *}$ and ${ }^{* * *}$ represent $\mathrm{p}<0.05, p<0.01$ and $p<0.001$ respectively (non-parametric Mann-Whitney test between dead and alive cells in individual genotypes and between indicated genotypes with $\mathrm{q}<0.05$ of Benjamini-Hochberg procedure). (d) Graphs showing the fraction of dead cells or cells that released IL-1 $\beta$ over time in BMDMs from mice with the indicated genotypes stimulated with $2 \mu \mathrm{g} / \mathrm{ml}$ of crude LPS. ${ }^{* * *}$ represent $p<0.001$ (Kaplan-Meier method with the log-rank test between indicated genotypes). (e) Dot-plot representation of the signal intensity of $\mathrm{IL}-1 \beta$ and $\mathrm{IL}-1 \alpha$ secreted from single cells, analysed in BMDMs with the indicated genotypes stimulated with $1 \mu \mathrm{g} / \mathrm{ml}$ of ultrapure LPS for 12 hours. The 
secretion signals of IL- $1 \alpha /$ IL- $1 \beta(+/+)$ cells in $\mathrm{A} 20^{\mathrm{MYC}-\mathrm{KO}}$ were moderately correlated with Pearson's $r$ $=0.68, p<0.001$. Data are representative of three independent experiments. All statistical tests are two-tailed. Raw data are provided in Supplementary Table 3 and unprocessed immunoblots are provided in Supplementary Figure 9.

Figure 7. ZnF7 is essential for A20-mediated inhibition of inflammation. (a) Graphs depicting the body weight (BW) of 9-week-old $A 20^{\mathrm{wt} / \mathrm{mZnF7}}$ and $A 20^{\mathrm{mZnF7} / \mathrm{mZnF7}}$ mice. (b) Graphs depicting spleen weight and spleen/body weight ratio in 28-week-old $A 20^{\text {wt/mZnF7 }}$ and $A 2 O^{\mathrm{mZnF7} / \mathrm{mZnF7}}$ littermate mice. Dots in the graphs indicate individual mice (male $A 20^{\mathrm{wt} / \mathrm{mZnF7}}, \mathrm{n}=12$; male $A 20^{\mathrm{mZnF} / \mathrm{mZnF7}}, \mathrm{n}=3$; female $A 20^{\mathrm{wt} / \mathrm{mZnF7}}, \mathrm{n}=8$ and female $A 20^{\mathrm{mZnF} 7 / \mathrm{mZnF7}}, \mathrm{n}=5$ mice for $\mathrm{a} ; A 20^{\mathrm{wt} / \mathrm{mZnF7}}, \mathrm{n}=8$ and $A 20^{\mathrm{mZnF} 7 / \mathrm{mZnF}}, \mathrm{n}=10$ mice for b). (c) Representative pictures of hindpaws of 28 -week-old $A 2 O^{\text {wt/mZnF7 }}$ and $A 2 O^{\mathrm{mZnF} 7 / \mathrm{mZnF7}}$ littermates, showing extensive swelling of toes in $A 20^{\mathrm{mZnF} / \mathrm{mZnF7}}$ mice. (d) Representative $\mathrm{mCT}$ pictures of the hindpaws of 28 -week-old $A 20^{\mathrm{wt} / \mathrm{mZnF7}}\left(\mathrm{n}=7\right.$ mice) and $A 20^{\mathrm{mZnF} 7 / \mathrm{mZnF7}}$ ( $\mathrm{n}=10$ mice) littermates in comparison to age-matched $A 2 O^{\mathrm{MYC}-\mathrm{KO}}$ mice. (e) Representative histological images of ankle joints from 28-week-old littermate mice with the indicated genotypes. Scale bar: $500 \mu \mathrm{m}$. (f-h) Graphs depicting histological scores for inflammation (f), bone erosion (g), and cartilage destruction (h) in mice with the indicated genotypes. Dots in the graphs indicate individual mice $\left(A 20^{\text {wt } / m Z n F 7}\right.$, $\mathrm{n}=8 ; A 20^{\mathrm{mZnF} 7 / \mathrm{mZnF7}}, \mathrm{n}=10$ mice for e-h). In all graphs mean \pm SEM is also shown for each group of mice. ${ }^{*},{ }^{* *}$ and ${ }^{* * *}$ represent $p<0.05, p<0.01$ and $p<0.001$ respectively (non-parametric MannWhitney test between indicated genotypes). All statistical tests are two-tailed. Raw data are provided in Supplementary Table 3.

Figure 8. $\mathrm{ZnF7}$ is critical for A20-mediated suppression of LPS-induced IL-1 $\beta$ and IL-1 $\alpha$ secretion and for the recruitment of A20 to the TNFR1 signalling complex. (a) IL $1 \alpha$ and IL1 $\beta$ protein levels in the supernatant of BMDMs from wild-type (WT, $n=10), A 20^{\mathrm{mZnF} / \mathrm{mZnF7}}(\mathrm{n}=10)$ and A20 ${ }^{\mathrm{MYC}-\mathrm{KO}}(\mathrm{KO}, \mathrm{n}=5)$ mice stimulated with LPS $(1 \mu \mathrm{g} / \mathrm{ml})$ for $6 \mathrm{~h}$. In all graphs mean \pm SEM is also shown for each group of mice. ${ }^{*},{ }^{* *}$ and ${ }^{* * *}$ represent $p<0.05, p<0.01$ and $p<0.001$ respectively (twoway ANOVA post-Bonferroni test between indicated genotypes). (b) Immunoblot showing pro-IL-1 $\beta$ $(31 \mathrm{kDa})$ and mature IL-1 $\beta(17 \mathrm{kDa})$ protein levels in the supernatant of BMDMs from wild-type (WT), $A 20^{\mathrm{mZnF} / \mathrm{mZnF7}}$ and $\mathrm{A} 20^{\mathrm{MYC}-\mathrm{KO}}$ mice stimulated with LPS $(1 \mu \mathrm{g} / \mathrm{ml})$ for $6 \mathrm{~h}$. (c) BMDMs from wild-type (WT), $A 20^{\mathrm{mZnF} 7 / \mathrm{mZnF7}}$ and $\mathrm{A} 20^{\mathrm{MYC}-\mathrm{KO}}$ mice were stimulated with Flag-TNF $(1 \mu \mathrm{g} / \mathrm{ml})$ for the indicated time points. Subsequently the TNF-RSC was immunoprecipitated using $\alpha$-Flag beads in the presence of USP2 $(24 \mu \mathrm{g} / \mathrm{ml})$ and $\lambda$ PPase $(8 \mathrm{U} / \mathrm{ul})$ and immunoblotted for A20, RIPK1, TRADD and TNFR1. (d) BMDMs from wild-type (WT), $A 20^{\mathrm{mZnF} / \mathrm{mZnF7}}$ and $\mathrm{A} 20^{\mathrm{MYC}-\mathrm{KO}}$ mice were stimulated with Flag-TNF $(1 \mu \mathrm{g} / \mathrm{ml})$ for the indicated time points, the TNF-RSC was immunoprecipitated using $\alpha-$ Flag beads and immunoblotted for RIPK1 and linear ubiquitin (M1) and TRADD. Data shown are representative of three independent experiments. All statistical tests are two-tailed. Raw data are provided in Supplementary Table 3. 